\title{
Late treatment with imatinib mesylate ameliorates radiation-induced lung fibrosis in a mouse model Minglun Li ${ }^{1}, 5$, Amir Abdollahi ${ }^{1,4}$, Hermann-Josef Gröne2, Kenneth E Lipson ${ }^{3,6}$, Claus Belka ${ }^{5}$ and Peter E Huber*1,4
}

Address: ${ }^{1}$ Department of Radiation Oncology German Cancer Research Center (DKFZ), Im Neuenheimer Feld 280, Heidelberg 69120, Germany, ${ }^{2}$ Molecular Pathology, German Cancer Research Center (DKFZ), Im Neuenheimer Feld 280, Heidelberg 69120, Germany, ${ }^{33} \mathrm{M}$ Pharmaceuticals, St. Paul, MN 55144, USA, ${ }^{4}$ Department of Radiation Oncology, University of Heidelberg Medical School, Heidelberg 69120, Germany, ${ }^{5}$ Department of Radiation Oncology, University of Munich medical school, Munich 81377, Germany and ${ }^{6}$ Fibrogen, Inc., South San Francisco, CA 94080, USA

Email: Minglun Li - minglun.li@med.uni-muenchen.de; Amir Abdollahi - a.amir@dkfz.de; Hermann-Josef Gröne - h.-j.groene@dkfz.de; Kenneth E Lipson - klipson@fibrogen.com; Claus Belka - Claus.Belka@med.uni-muenchen.de; Peter E Huber* - p.huber@dkfz.de

* Corresponding author

Published: 21 December 2009

Radiation Oncology 2009, 4:66 doi:I0.1 I86/I748-7|7X-4-66

This article is available from: http://www.ro-journal.com/content/4/I/66

(C) 2009 Li et al; licensee BioMed Central Ltd.

This is an Open Access article distributed under the terms of the Creative Commons Attribution License (http://creativecommons.org/licenses/by/2.0), which permits unrestricted use, distribution, and reproduction in any medium, provided the original work is properly cited.

\begin{abstract}
Background: We have previously shown that small molecule PDGF receptor tyrosine kinase inhibitors (RTKI) can drastically attenuate radiation-induced pulmonary fibrosis if the drug administration starts at the time of radiation during acute inflammation with present but limited effects against acute inflammation. To rule out interactions of the drug with acute inflammation, we investigated here in an interventive trial if a later drug administration start at a time when the acute inflammation has subsided - has also beneficial antifibrotic effects.
\end{abstract}

Methods: Whole thoraces of C57BL/6 mice were irradiated with $20 \mathrm{~Gy}$ and treated with the RTKI imatinib starting either 3 days after radiation (during acute inflammation) or two weeks after radiation (after the acute inflammation has subsided as demonstrated by leucocyte count). Lungs were monitored and analyzed by clinical, histological and in vivo non-invasive computed tomography as a quantitative measure for lung density and lung fibrosis.

Results: Irradiation induced severe lung fibrosis resulting in markedly reduced mouse survival vs. non-irradiated controls. Both early start of imatinib treatment during inflammation and late imatinib start markedly attenuated the development of pulmonary fibrosis as demonstrated by clinical, histological and qualitative and quantitative computed tomography results such as reduced lung density. Both administration schedules resulted in prolonged lifespans. The earlier drug treatment start resulted in slightly stronger beneficial antifibrotic effects along all measured endpoints than the later start.

Conclusions: Our findings show that imatinib, even when administered after the acute inflammation has subsided, attenuates radiation-induced lung fibrosis in mice. Our data also indicate that the fibrotic fate is not only determined by the early inflammatory events but rather a complex process in which secondary events at later time points are important. Because of the clinical availability of imatinib or similar compounds, a meaningful attenuation of radiation-induced lung fibrosis in patients seems possible. 


\section{Background}

Radiotherapy is a mainstay of treating neoplasm in lungs [1-4]. Fibrosis is the main chronic side effect of radiotherapy that potentially prevents to deliver the necessary dose to benefit cancer patients [5-7]. Although modern techniques of radiotherapy (stereotactic radiotherapy, intraoperative radiotherapy, interstitial brachytherapy etc.) are now increasingly used to improve dose distribution and reduce side effects, radiation induced fibrotic lesions still occur [8-10]. There has been remarkably little progress in the development of effective antifibrotic therapies $[7,11]$.

Recent studies indicated that pulmonary fibrosis is not a unique pathologic process but rather an excess of the same biologic events involved in normal tissue repair with persistent and exaggerated wound healing ultimately leading to an excess of fibroblast replication and matrix deposition $[7,11]$. A cascade of many cytokines leading to lung fibrosis after radiation injury has been described $[12,13]$. Recently, a number of new regulators in radiation-induced lung injury such as intercellular adhesion molecules (ICAM-1) and the CD95 ligand system have been reported $[14,15]$. Typical fibrogenic mediators include TGF- $\beta$, IL- 1 , TNF- $\alpha$, bFGF, and thrombin, but also PDGF has been implicated to demonstrate profibrotic activities [16-21]. Thus the PDGF/PDGFR system can be considered as a promising target for treating fibrotic diseases [22-26].

Recently, we have shown that PDGF receptor tyrosine kinase inhibitors (RTKI) including imatinib can attenuate radiation-induced pulmonary fibrosis if the drug administration starts before the toxic event or within three days after the insult [27]. PDGF RTKI prolonged survival and protected mice from lung fibrosis, presented as reduced lung density measured by computed tomography examinations, although the radiation-induced acute inflammation was not significantly abrogated. Thus we hypothesized that fibrogenesis is a separate process after acute inflammation, correlated but not dependent to acute inflammation [27].

However in this previous study PDGF RTKI (SU9518) was administrated during RT-induced acute inflammation (1 day before and 3 days after radiation) showing no marked but certain effects on the acute inflammation. Therefore, acute inflammation is affected to some extent by concurrent drug administration and, even if the measurable extent of inflammation had not been significantly affected, the administration during inflammation could still be a prerequisite for the later antifibrotic effects. Thus, in the present study we chose a late drug treatment starting at the time when the primary inflammation has subsided, to rule out direct and indirect effects associated with acute inflammation,
Therefore we report here the full results of the imatinib experiments including the data on the late imatinib administration arm starting two weeks after radiation, at a time when the acute inflammation has already completely subsided. These experiments thus investigate i) the role of acute inflammation in the development of radiation induced lung fibrosis, ii) if attenuation of the acute inflammation is necessary to block fibrosis and iii) if a late drug administration after radiation insult and after the acute inflammation has subside still has antifibrotic potential. To this end thorax of C57BL/ 6 mice were irradiated with $20 \mathrm{~Gy}$ and mice were subsequently treated with imatinib mesylate/Gleevec starting either three days after radiation or two weeks after radiation. Longitudinal follow-up of the mice lungs in vivo was performed by noninvasive radiological monitoring using high resolution computed-tomography [28].

\section{Methods \\ Experimental protocol and animal model}

All animal procedures were approved by institutional and governmental authorities (Regierungspraesidium Karlsruhe, Germany). Fibrosis-prone mice (female C57BL/6J, 8 weeks old, approximate body weight 20 g, Charles River Laboratories, Sulzfeld, Germany) were used. For thoracic irradiation, mice were anesthetized by intraperitoneal application of Domitor (Pfizer, Exton, USA; $0.2 \mathrm{mg} / \mathrm{kg}$ ) and Ketamin 10\% (Park, Davis \& Company, Berlin, Germany; $100 \mathrm{mg} / \mathrm{kg}$ ). Cobalt-60 gamma radiation (Siemens Gammatron S, Erlangen, Germany) was administered as single dose $(20 \mathrm{~Gy})$ to the entire thorax $(0.441 \mathrm{~Gy} / \mathrm{min}$; source surface distance: $0.7 \mathrm{~m}$ ) using one standing field anterior-posterior. Other organs, above and beyond the thorax were shielded. Animals were supplied with diet and water ad libitum.

Imatinib Mesylate was provided by SUGEN Inc. South San Francisco, CA. To achieve clinically relevant doses $[26,27]$, Imatinib were formulated in standard mouse chow at 0.5 $\mathrm{mg} / \mathrm{g}$ resulting in a dosage of $40 \mathrm{mg} / \mathrm{kg} / \mathrm{d}$. Imatinib (Gleevec) is known to have high activity against three kinases: Bcr/Abl, c-Kit, and PDGFR- $\alpha$ and $-\beta[26,27]$. In irradiated animals, imatinib treatment was either started three days after radiation or two weeks after radiation and was continued until the end of observation, as stated in our previous study [27]. Animals were checked three times weekly, clinically examined and weighed.

\section{Lung histology}

Histological analysis from mice tissues was performed systematically at early and later time points after radiation as described $[27,28]$. Briefly, lungs were fixed by intratracheal instillation of $4 \%$ formalin, followed by overnight fixation, embedding in paraffin, sectioned at $5 \mu \mathrm{m}$, and stained with hematoxylin-eosin (H\&E). The total count of 
leukocytes was determined by morphometric evaluation (Q 600 Quantimet, Leica Microsystems, Wetzlar, Germany) and the septal thickness was measured in 5 regions of interest (ROI) for each mouse.

\section{High-resolution computed tomography (HRCT) of mouse lungs}

To obtain an independent qualitative and quantitative measure for lung fibrosis in the mice we used high-resolution computed tomography (CT). CT is the method of choice for monitoring fibrosis in patients. This radiological method allows non-invasive and repeated measurements in the same mice in a longitudinal way [28]. CT exams were performed in 5 randomly selected mice from each group every second week during the entire observation period. CT images were captured on a Toshiba multislice CT scanner (Aquilion 32). $120 \mathrm{kV}$ with $100 \mathrm{mAS}$ were applied. $0.5 \mathrm{~mm}$ thin slices with $0.5 \mathrm{~mm}$ inter-slice distance spanned the complete mouse chest (total acquisition time 0.5 seconds). Multiplanar reconstructions (MPR) were performed for semiquantitative analysis. Hounsfield units (HU) of section slides from the upper and lower lung region were determined. Eight regions of interest (ROI) were defined in the following areas: the right upper anterior and posterior regions, the left upper anterior and posterior regions, the right lower anterior and posterior regions and the left lower anterior and pos- terior regions. Total arithmetic means $\pm \mathrm{SE}$ of the HU were calculated.

\section{Statistics}

Mouse survival curves after thoracic irradiation and imatinib treatments were calculated with the Kaplan-Meier method and compared using the log-rank test. Other quantitative data are given as mean values \pm SD or as indicated. For analysis of differences between the groups, ANOVA followed by the appropriate post hoc test for individual comparisons between the groups was performed. All tests were two-tailed. $P<0.05$ was considered statistically significant.

\section{Results \\ Late imatinib interventive treatment prolongs mouse survival}

A single dose of 20 Gy thoracic irradiation induced marked lung fibrosis and dramatically reduced animal survival versus nonirradiated animals. Median survival was 19 weeks after radiation $v$ s. control mice which stayed alive for more than one year $(P<0.0001)$. The KaplanMeier curves are depicted in figure 1a. Imatinib treatment starting $3 \mathrm{~d}$ after radiation prolonged median survival by $\sim 11$ weeks with a median survival of 30 weeks $(P<0.01$ vs. radiation alone) as shown in a previous study [27]. Importantly to us, imatinib treatment starting 2 weeks (a)

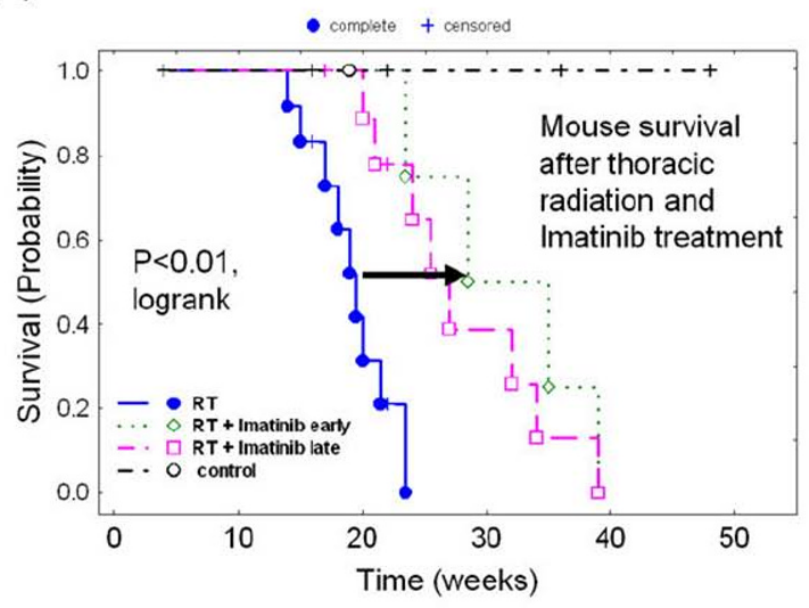

(b)

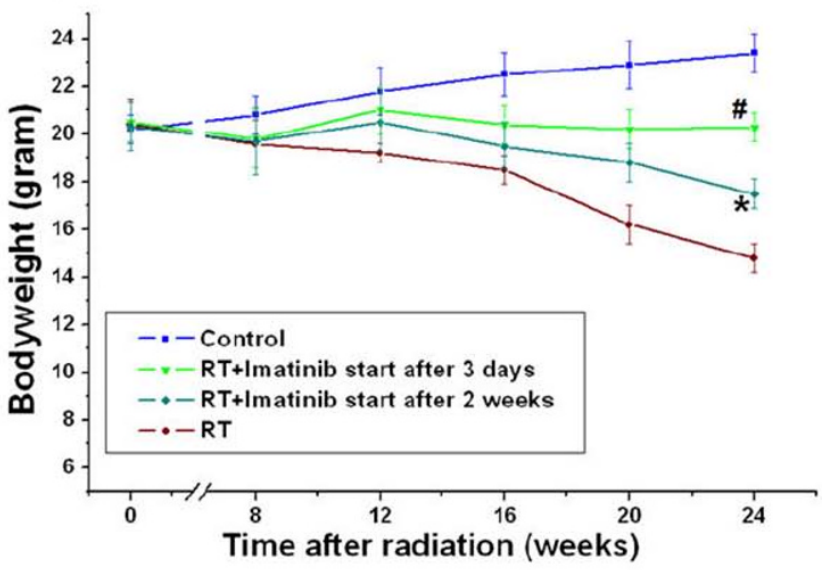

Figure I

(a) Kaplan-Meier analysis of mouse survival following thoracic irradiation and imatinib treatment. Death was considered complete (cause-specific due to radiation) in all cases except those of planned euthanasia for histological assessment, which were considered as censored. Radiation $(20 \mathrm{~Gy})$ reduced survival $(P<0.00 \mathrm{I}$ vs. the control as reported in $(24))$. Imatinib treatment increased mouse survival if administration started as late as 2 weeks after radiation ( $P<0.02$ vs. radiation) and if started early within 3 days after radiation $(P<0.01$ as reported in $(24))$. The earlier start of drug treatment tended to be more effective in prolonging survival than later start of drug treatment, but this difference was not significant $(P>0.1)$. (b) Bodyweight follow-up after thoracic irradiation and imatinib treatment. Five mice were randomly selected in each group and weighed every two weeks. Mean $\pm \mathrm{SE}$ was presented. $* \mathrm{P}<0.0 \mathrm{I}$ vs. the $\mathrm{RT}$ only group; \# $\mathrm{P}<0.0 \mathrm{I}$ vs. the control group. 
after radiation also prolonged median survival by $\sim 8$ weeks with a median survival of 27 weeks $(P<0.02$ vs. radiation alone). The difference in survival between earlier and later treatment schedules was not statistically significant $(P>0.1)$, but a tendency was present suggesting that the earlier drug treatment start was beneficial.

To support the survival data we also analyzed the animals' clinical status. We found that imatinib treatment in both early and late treatment arms also attenuated radiationrelated clinical adverse effects such as weight loss (figure $1 \mathrm{~b}, \mathrm{P}<0.02$, at all time points after week 14 ) and other clinical parameters, which were monitored weekly over the entire observation period. In specific, imatinib early and late improved clinical status including animal behavior (worse after irradiation, improved by imatinib), tachypnoea and heart rate (both higher after irradiation, reduced by imatinib). Again, a marked difference of the benefits caused by imatinib in the clinical animal parameters between the two imatinib schedules was not observed.

\section{Computed tomography of mice lungs}

Computed tomography (CT) was used to obtain an independent qualitative and quantitative measure of mice lung fibrosis that could be repeated in the same animal over time. As reported before [24], after week 16 typical radiological features of lung fibrosis were visible after 20 Gy irradiation including irregular septal thickening, patchy peripheral reticular abnormalities with intralobular linear opacities and subpleural honeycombing (figure $2 \mathrm{a})$. The extent of fibrotic disease progression in CT images correlated well with histology and clinical impairment. Imatinib treatment was able to markedly reduce the radiological/morphological signs of fibrosis after radiation in both early and late imatinib treated mice. In addition to the morphological assessment, CT enabled quantitation of fibrosis by an assessment of the lung density (quantified in Hounsfield units (HU)). We found that lung density drastically increased during weeks 12 to 24 post radiotherapy (figure $2 \mathrm{~b}$ ) in irradiated mice only. Imatinib in both early and late application arms strongly inhibited this increase by approximately $50 \%(\mathrm{P}<0.001)$. The earlier therapy start appeared to be slightly more effective in reducing CT signs of lung fibrosis than a later therapy, but this tendency did not reach statistical significance $(P>0.1)$.

\section{Histological assessment of lung fibrosis after irradiation}

It is assumed that exposure of normal lung tissue to irradiation has two well-recognized adverse effects: acute/ subacute pneumonitis and fibrosis as long term sequelae

(a)

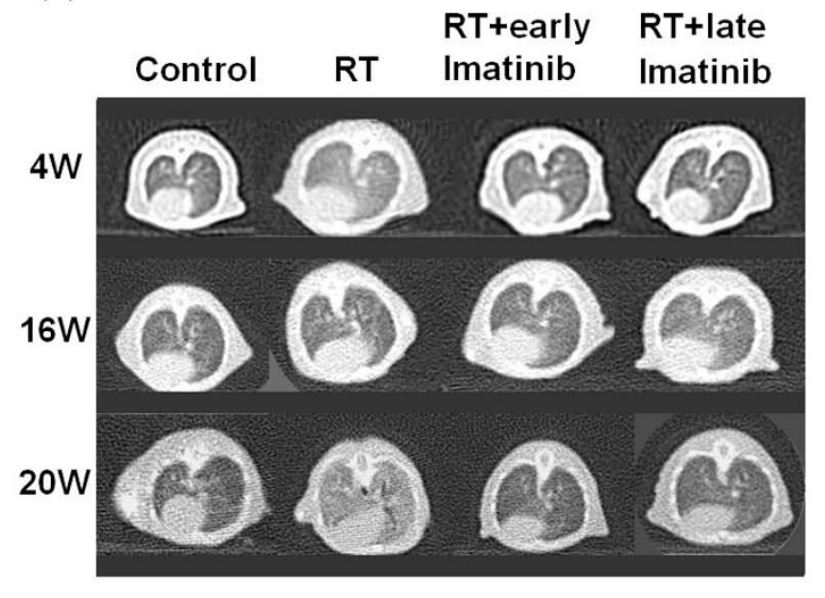

(b)

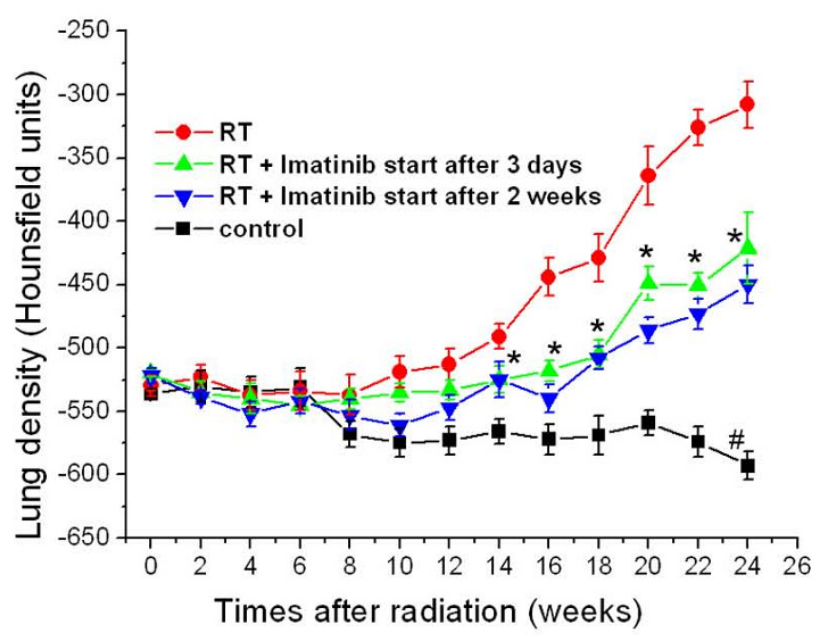

Figure 2

(a) High resolution computed tomography (CT) as a non-invasive tool for qualitative and quantitative longitudinal monitoring of pulmonary fibrosis progression in mice. Representative CT scans showing progression of pulmonary fibrosis in mice after 20 Gy whole thorax irradiation (RT), and treatment with imatinib treatment starting 3 days and 2 weeks after radiation (RT). Fibrosis is characterized by diffuse bilateral areas of "ground-glass" attenuation and intralobular reticular opacities. (b) Quantitative lung density values derived from CT scans. The same 5 randomly chosen mice in each treatment group were examined in a longitudinal study by CT every 2 weeks. 8 regions of interest (ROI) were randomly selected in the lungs and the lung density (in Hounsfield Units (HU)) was determined for each ROI. Mean \pm SE are presented. * $\mathrm{P}<0.0$ I vs. the RT only group; \# $\mathrm{P}<0.01$ vs. the control group. 
[11,27-29]. To better understand the pathogenesis of the radiation-induced lung fibrosis process, and to evaluate the modulation after radiation and imatinib, mice were selected for analysis of leukocyte infiltration, edema and collagen-deposition with associated thickening of the alveolar septum.

As described earlier a biphasic radiation response was observed, initially consisting of acute and subacute pneumonitis, which was followed by the onset of fibrogenesis [27]. The characteristic histologic findings in the pneumonitis phase of the radiation response were prominent inflammatory cell infiltrates in the alveoli and lung interstitium with simultaneous interstitial edema (figure 3a). Both parameters exhibited similar kinetics in the acute phase, reaching their maxima about 72 hours after radiation injury. After the acute radiation response, leukocyte count spontaneously subsided within one week (figure 4a). When imatinib administration started early during inflammation the treatment did not markedly decrease this first, radiation-induced acute leukocyte peak ( $\mathrm{P}>$ $0.2)$, although imatinib affected the inflammation to some extent and, the tendency was seen that imatinib had a nonsignificant tendency to reduce acute inflammation in terms of reduced edema and leucocyte count.

Histological analysis of irradiated lungs further showed the development of fibrosis by progressive collagen deposition after week 12 (figure 3b). This fibrogenesis phase was characterized by development of typical fibroblast foci and exuberant deposition of extracellular matrix in irradiated lungs (figure 3c). Both imatinib schedules reduced collagen deposition and septal thickness (figure $4 \mathrm{~b}$ ), while the early administration appeared to be slightly more effective than late administration. In irradiated mice, the later fibrogenesis phase was accompanied by a strong second onset of leukocyte infiltration that began several weeks after irradiation and reached a peak at approximately 20 weeks post irradiation.

At later time points ( $>20$ weeks) the fibrotic foci evolved and coalesced into widespread fibrosis with remodeling of the lung architecture. Moreover, in the irradiated lungs the second onset of progressive fibrosis-related leukocyte infiltration persisted until the morphologically described fibrosis process was completed (after week 26). Figure 4a also shows that this second inflammatory response was also reduced in terms of reduced leucocyte count by both early and late imatinib treatment $(\mathrm{P}<0.05)$. Here again, the earlier drug treatment start tended to be slightly more effective than the later treatment start, but this difference was not statistically significant $(\mathrm{P}>0.5)$.

\section{Discussion}

Here we confirm that imatinib $\left(\right.$ Gleevec $^{\circledR}$ ) treatment is an effective strategy to attenuate radiation-induced lung fibrosis in mice. The beneficial drug effects are present even when drug administration starts two weeks after radiation when the acute radiation associated inflammation is completely subsided. The antifibrotic drug effectivity after the radiation-induced inflammation suggests that the fibrotic fate after radiation is not completely determined by the early inflammatory events, but rather by complex secondary signalling processes [27-31]. Therefore the present paper confirms and extends previous publications on imatinib showing beneficial antifibrotic effects in a radiation induced lung fibrosis model if the drug treatment starts before or after the radiation insult but within the acute inflammation [27].

Both early and late drug treatment start were able to attenuate the development of radiation induced lung fibrosis as shown by histological analysis during the relative long time course of lung fibrogenesis of up to 26 weeks. Imatinib markedly attenuated the development of fibroblast foci and the subsequent remodeling of the lung architecture. The morphological beneficial effects of imatinib were in agreement with qualitative and quantitative highresolution computed tomography scans of mouse lungs. Moreover, a significant survival benefit and reduced clinical morbidity in imatinib treated mice was seen for both treatment schedules.

When comparing the earlier ( 3 days) $v$ s. the later imatinib treatment start ( 2 weeks after radiation) we found that the earlier therapy start was beneficial with respect to all endpoints tested (histology, survival, CT monitoring, clinical behaviour), but this advantageous tendency for early treatment did not reach statistical significance.

One assumption of the inflammation and fibrosis debates were that fibrosis could be avoided if the early inflammation cascade was interrupted before irreversible tissue injury occurred [7]. However, early anti-inflammatory therapies, even in combination with potent immunosuppressives, fail to improve the disease outcome in the clinical setting $[7,11]$. Therefore, acute inflammation is probably not the only critical step in the development of the fibrotic response. In our setting, although neither the early imatinib start markedly reduced the acute inflammatory response nor the later start did (which was scheduled on purpose to not interfere with acute inflammation), the drug still attenuated the onset and development of lung fibrosis. Conversely, the second inflammatory response occurring around 12 weeks and later after radiation, was dramatically attenuated by both imatinib schedules. Thus the inhibition of the later fibrogenesis consisting of stromal cell migration, proliferation and extracellular matrix deposition seems to be a principal drug target.

A viable hypothesis is that imatinib's antifibrotic effects in the radiation lung model are conveyed via inhibition of 


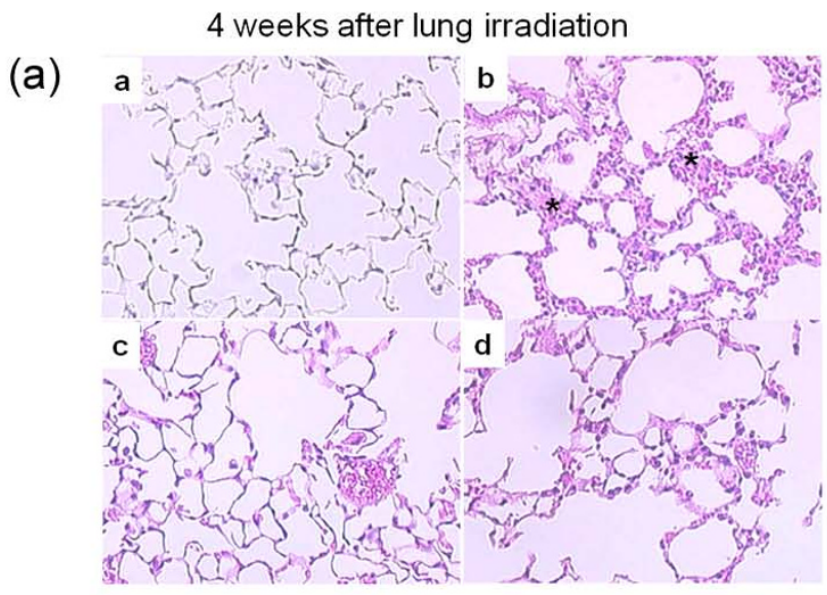

(b)

16 weeks after lung irradiation

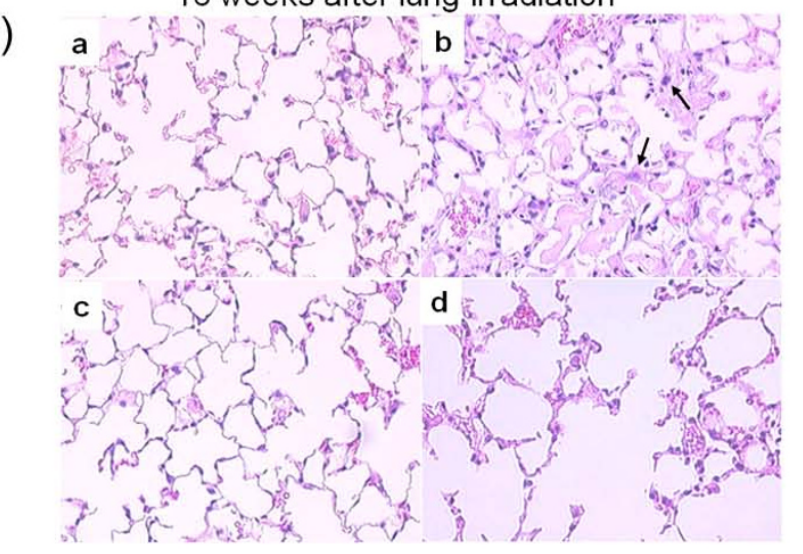

\section{0 weeks after lung irradiation}

(c)

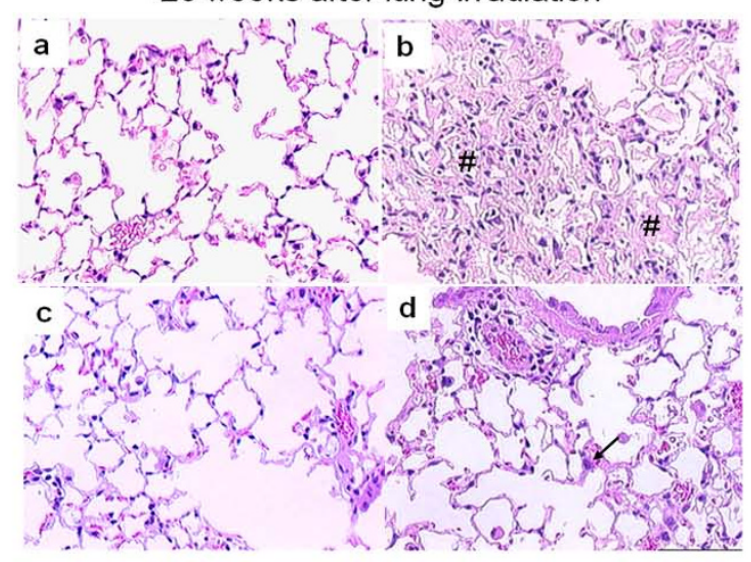

\section{Figure 3}

(a) Photomicrographs of H\&E stained mouse lung tissue sections from a) control mice, b) irradiated mice (20 Gy, RT) and mice treated with imatinib starting 3 days (c) or 2 weeks (d) after thoracic irradiation. Leukocytes infiltration was marked with asterisk. (b) Photomicrographs of H\&E stained mouse lung tissue sections at 16 weeks from a) control mice, b) irradiated mice $(20 \mathrm{~Gy}, \mathrm{RT})$ and mice treated with imatinib starting 3 days (c) or 2 weeks (d) after thoracic irradiation. Fibroblasts were marked with arrow. (c) Photomicrographs of H\&E stained lung tissue sections at 20 weeks from a) control mice, b) irradiated mice (20 Gy, RT) and mice treated with imatinib starting 3 days (c) or 2 weeks (d) after thoracic irradiation. Collagen depositions were marked with \#. 
(a)

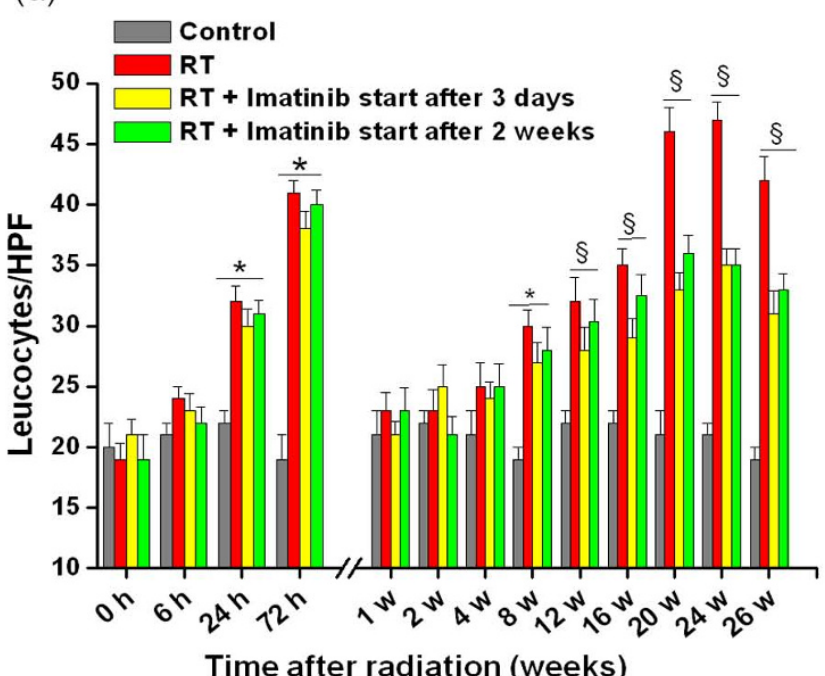

(b)

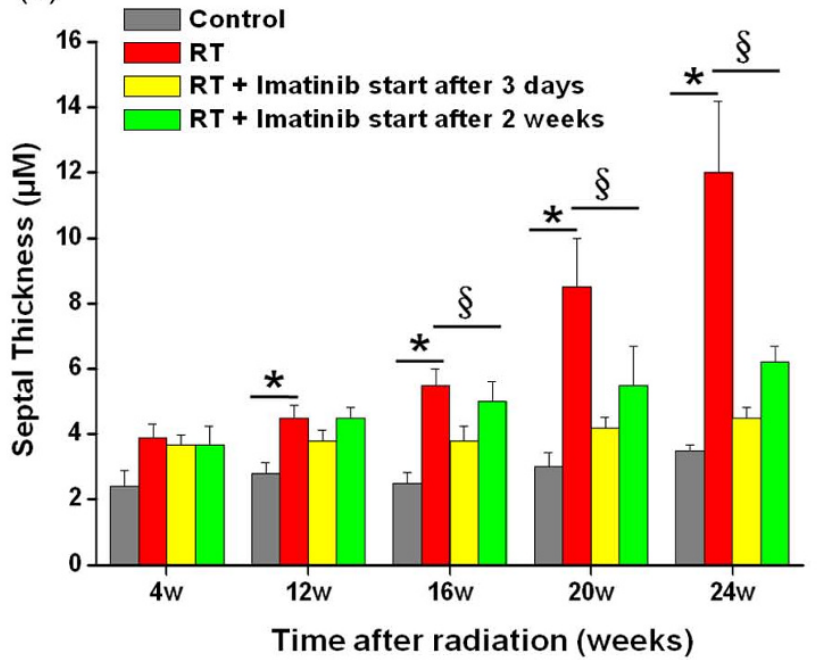

Figure 4

(a) Quantitative analysis of leukocyte numbers as inflammation parameter. Bars are mean $\pm \mathrm{SE}$. * $p<0.05$ vs. controls. §p $<0.05$ vs. radiation only for both early and late imatinib schedules. (b) Quantitative analysis of septal thickness as fibrotic parameter presenting deposition of extracellular collagen. Bars are mean $\pm \mathrm{SE}$. ${ }^{*} \mathrm{p}<0.05$ vs. controls. $\S \mathrm{p}<0.05$ vs. radiation only, for both early and late imatinib schedules.

PDGF signalling, although imatinib has been demonstrated to show marked activity against at least three kinases: Bcr/Abl, c-Kit, PDGFR- $\alpha$ and $-\beta$ which can all be linked to fibrosis, in particular in conjunction with TGF- $\beta$ signalling [22-24]. The potential role of PDGF signalling for the development of lung fibrosis, and in turn for the treatment of fibrosis by inhibiting PDGF signalling is supported by data in idiopathic pulmonary fibrosis, asbestos, bleomycin- and radiation-induced lung fibrosis as well as in fibrosis in other organs such as the kidneys, liver, skin and heart [16-18,23-26]. Therefore, together with data on radiation induced PDGF expression and phosphorylation of PDGFR in vitro and in vivo, and the inhibition by the kinase inhibitors, we believe that the inhibition of PDGFR signalling is a key mechanism behind our functional findings $[27,30]$.

Nevertheless, one cannot exclude important primary roles of Bcr/Abl, c-Kit, or TGF-beta pathways and one should also keep in mind that ATP-competitive kinase inhibitors rarely exhibit complete selectivity. Therefore many additional data towards a better mechanistic understanding of our data could be obtained. At the same time, it will be difficult to proof that one or more specific kinase/kinases resulting in one or several protein expression event and no other cascade is responsible for the beneficial role of imatinib here. For example, although others and we had previously shown that PDGF RTKI inhibited phosphorylation of PDGFR in vivo and this likely contributed to the bene- fits, it has also been reported that imatinib's c-kit effects and its link to TGF-beta might be the responsible beneficial antifibrotic pathway [22].

Moreover, the data should also be interpreted with the understanding that there may also be additional potential off-target effects. Such off-target effects may have well contributed to the antifibrotic effects that these compounds have. Rather than pointing out a single protein or gene, it is conceivable that the development of fibrosis is not a single step event, but rather an imbalance of an otherwise physiological homeostatic system with many players involved. In these terms fibrogenesis may be described as a shift of the homeostatic system towards the profibrotic state with the consequence that the entire process can only be understood as a gene and protein network shift which may call for systematic biology approaches for a deeper and more correct understanding [32-34].

The network idea is perhaps fostered by the fact that e.g. PDGF signalling alone is not an exclusive feature of fibrosis research but also known as a key signalling in cancer research, since PDGF signalling is considered to be a driving force for cancer cells and known to be proangiogenic [35]. Accordingly, the inhibition of PDGF signalling is being investigated as anticancer drugs alone and in combination with chemotherapy and radiotherapy [36-40]. Therefore, a two-fold rationale for the use of PDGF RTKI in radiation oncology might unfold: first, employing the 
anticancer effects of PDGF inhibition while second, simultaneously decreasing fibrosis as a common adverse side effect in radiotherapy [41,42]. However, again, it is unlikely that single pathway inhibition can completely prevent lung fibrosis, considering the intricate genetic networking associated with this complex process. While our data indicate that fibrosis can be attenuated or delayed, it still progressed despite imatinib. Therefore, RTKI should be considered in the context of other drug therapies, especially since, as for any other drug, potential side effects e.g. cardiotoxicity have been reported for imatinib [43].

\section{Conclusions}

Taken together, we demonstrate here that drug treatment using imatinib might be a useful therapeutic approach to attenuate radiation-induced lung fibrosis or other types of fibrosis, which exhibits benefits even after the damaging insult and its acute inflammation has completely subsided.

\section{Competing interests}

The authors declare that they have no competing interests.

\section{Authors' contributions}

ML performed experiments, analyzed data and participated in writing the manuscript. AA participated in designing the study and analyzed data. KEL participated in the study design and manuscript writing. HJG performed and analyzed histology. PEH designed the study, analyzed data, and wrote the manuscript. All authors approved the final version of the manuscript.

\section{Acknowledgements}

We would like to thank Alexandra Tietz and Peter Peschke PhD. This work was supported in part by grants from the Deutsche Krebshilfe 106997, DFG National Priority Research Program the tumor-vessel interface (SPPI 190) and NASA/NSCOR NNJ04HJI2G, the Tumorzentum Heidelberg-Mannheim, and BMBF 03NUK004A-C.

\section{References}

I. Hurkmans CW, Cuijpers JP, Lagerwaard FJ, Widder J, Heide UA van der, Schuring D, Senan S: Recommendations for implementing stereotactic radiotherapy in peripheral stage IA non-small cell lung cancer: report from the Quality Assurance Working Party of the randomised phase III ROSEL study. Radiat Oncol 2009, 4:I.

2. Eckert F, Mueller AC: SCLC extensive disease - treatment guidance by extent or/and biology of response? Radiat Oncol 2008, 3:33.

3. Uitterhoeve AL, Koolen MG, van Os RM, Koedooder K, Kar M van de, Pieters BR, Koning CC: Accelerated high-dose radiotherapy alone or combined with either concomitant or sequential chemotherapy; treatments of choice in patients with NonSmall Cell Lung Cancer. Radiat Oncol 2007, 2:27.

4. Collins BT, Erickson K, Reichner CA, Collins SP, Gagnon GJ, Dieterich S, McRae DA, Zhang Y, Yousefi S, Levy E, Chang T, Jamis-Dow C, Banovac F, Anderson ED: Radical stereotactic radiosurgery with real-time tumor motion tracking in the treatment of small peripheral lung tumors. Radiat Oncol 2007, 2:39.

5. Milano MT, Constine LS, Okunieff P: Normal tissue toxicity after small field hypofractionated stereotactic body radiation. Radiat Oncol 2008, 3:36.
6. Yamashita H, Nakagawa K, Nakamura N, Koyanagi H, Tago M, Igaki $\mathrm{H}$, Shiraishi $\mathrm{K}$, Sasano N, Ohtomo K: Exceptionally high incidence of symptomatic grade 2-5 radiation pneumonitis after stereotactic radiation therapy for lung tumors. Radiat Oncol 2007, 2:21.

7. Kamp DW: Idiopathic pulmonary fibrosis: the inflammation hypothesis revisited. Chest 2003, I 24: I I87-I 190.

8. Niew Niewald M, Fleckenstein J, Licht N, Bleuzen C, Ruebe C: Intraoperative radiotherapy (IORT) combined with external beam radiotherapy (EBRT) for soft-tissue sarcomas - a retrospective evaluation of the Homburg experience in the years 1995-2007. Radiat Oncol 2009, 4:32.

9. Viani GA, Novaes PE, Jacinto AA, Antonelli CB, Pellizzon AC, Saito EY, Salvajoli JV: High-dose-rate brachytherapy for soft tissue sarcoma in children: a single institution experience. Radiat Oncol 2008, 3:9.

10. Cornelissen R, Senan S, Antonisse IE, Liem H, Tan YK, Rudolphus A, Aerts JG: Bronchiolitis obliterans organizing pneumonia (BOOP) after thoracic radiotherapy for breast carcinoma. Radiat Oncol 2007, 2:2.

II. Mason RJ, Schwarz MI, Hunninghake GW, Musson RA: NHLBI Workshop Summary. Pharmacological therapy for idiopathic pulmonary fibrosis. Past, present, and future. Am J Respir Crit Care Med 1999, 160:177I-I777.

12. McBride WH: Cytokine cascades in late normal tissue radiation responses. Int J Radiat Oncol Biol Phys 1995, 33:233-234.

13. Hill RP, Rodemann HP, Hendry JH, Roberts SA, Anscher MS: Normal tissue radiobiology: from the laboratory to the clinic. Int J Radiat Oncol Biol Phys 200I, 49(2):353-65.

14. Heinzelmann F, Jendrossek V, Lauber K, Nowak K, Eldh T, Boras R, Handrick R, Henkel M, Martin C, Uhlig S, Köhler D, Eltzschig HK, Wehrmann M, Budach W, Belka C: Irradiation-induced pneumonitis mediated by the CD95/CD95-ligand system. J Natl Cancer Inst 2006, 98( ( 7): |248-5|.

15. Hallahan DE, Geng L, Shyr YJ: Effects of intercellular adhesion molecule I (ICAM-I) null mutation on radiation-induced pulmonary fibrosis and respiratory insufficiency in mice. J Natl Cancer Inst 2002, 94(10):733-4I.

16. Ostman A, Heldin CH: Involvement of platelet-derived growth factor in disease: development of specific antagonists. Adv Cancer Res 200I, 80: I-38.

17. Rice AB, Moomaw CR, Morgan DL, Bonner JC: Specific inhibitors of platelet-derived growth factor or epidermal growth factor receptor tyrosine kinase reduce pulmonary fibrosis in rats. Am J Pathol 1999, I55:213-22I.

18. Hasselbalch HC, Bjerrum OW, Jensen BA, Clausen NT, Hansen PB, Birgens $\mathrm{H}$, Therkildsen $\mathrm{MH}$, Ralfkiaer $\mathrm{E}$, et al.: Imatinib mesylate in idiopathic and postpolycythemic myelofibrosis. Am J Hematol 2003, 74:238-242.

19. Kumar S, Kolozsvary A, Kohl R, Lu M, Brown S, Kim JH: Radiationinduced skin injury in the animal model of scleroderma: implications for post-radiotherapy fibrosis. Radiat Oncol 2008, 3:40.

20. Bonner JC: Regulation of PDGF and its receptors in fibrotic diseases. Cytokine Growth Factor Rev 2004, 15:255-273.

21. Simone NL, Soule BP, Gerber L, Augustine E, Smith S, Altemus RM, Mitchell JB, Camphausen KA: Oral Pirfenidone in patients with chronic fibrosis resulting from radiotherapy: a pilot study. Radiat Oncol 2007, 2:19.

22. Daniels CE, Wilkes MC, Edens M, Kottom TJ, Murphy SJ, Limper AH, Leof EB: Imatinib mesylate inhibits the profibrogenic activity of TGF-beta and prevents bleomycin-mediated lung fibrosis. J Clin Invest 2004, I I 4: I 308-1316.

23. Vuorinen K, Gao F, Oury TD, Kinnula VL, Myllärniemi M: Imatinib mesylate inhibits fibrogenesis in asbestos-induced interstitial pneumonia. Exp Lung Res 2007, 33:357-73.

24. Azuma M, Nishioka $Y$, Aono $Y$, Inayama M, Makino H, Kishi J, Shono M, Kinoshita K, Uehara H, Ogushi F, Izumi K, Sone S: Role of alpha Iacid glycoprotein in therapeutic antifibrotic effects of imatinib with macrolides in mice. Am J Respir Crit Care Med 2007, 176(12): 1243-50.

25. Chaudhary NI, Roth G], Hilberg F, Müller-Quernheim J, Prasse A, Zissel G, Schnapp A, Park JE: Inhibition of PDGF, VEGF and FGF signalling attenuates fibrosis. Eur Respir J 2007, 29(5):976-85.

26. Yamasaki Y, Miyoshi K, Oda N, Watanabe M, Miyake H, Chan J, Wang $X$, Sun L, Tang C, McMahon G, Lipson KE: Weekly dosing with the 
platelet-derived growth factor receptor tyrosine kinase inhibitor SU95 I 8 significantly inhibits arterial stenosis. Circ Res 2001, 88:630-636.

27. Abdollahi A, Li M, Ping G, Plathow C, Domhan S, Kiessling F, Lee LB, McMahon G, Gröne HJ, Lipson KE, Huber PE: Inhibition of platelet derived growth factor (PDGF) signaling attenuates pulmonary fibrosis. J Exp Med 2005, 201:925-935.

28. Plathow C, Li M, Gong P, Zieher H, Kiessling F, Peschke P, Kauczor HU, Abdollahi A, Huber PE: Computed Tomography Monitoring of Radiation-Induced Lung Fibrosis in Mice. Invest Radiol 2004, 39:600-609.

29. Rubin P, Johnston CJ, Williams JP, McDonald S, Finkelstein JN: A perpetual cascade of cytokines postirradiation leads to pulmonary fibrosis. Int J Radiat Oncol Biol Phys 1995, 33:99-109.

30. Li M, Gong P, Plathow C, Trinh T, Lipson KE, Hauser K, Krempien R, Debus J, Abdollahi A, Huber PE: Small molecule receptor tyrosine kinase inhibitor of platelet-derived growth factor signaling (SU95 I 8) modifies radiation response in fibroblasts and endothelial cells. BMC Cancer 2006, 24;6(I):79.

31. Li M, Jendrossek $V$, Belka $C$ : The role of PDGF in radiation oncology. Radiat Oncol 2007, 2:5.

32. Huber PE, Hauser K, Abdollahi A: Genome Wide Expression Profiling of Angiogenic Signaling and the Heisenberg Uncertainty Principle. Cell Cycle 2004, 3: I348-I35 I.

33. Abdollahi A, Hahnfeldt P, Maercker C, Gröne HJ, Debus J, Ansorge W, Folkman J, Hlatky L, Huber PE: Endostatin's antiangiogenic signaling network. Mol Cell 2004, I 3:649-663.

34. Abdollahi A, Schwager C, Kleeff J, Esposito I, Domhan S, Peschke P, Hauser K, Hahnfeldt P, Hlatky L, Debus J, Peters JM, Friess H, Folkman J, Huber PE: Transcriptional network governing the angiogenic switch in human pancreatic carcinoma. PNAS 2007, 104: | 2890-12895.

35. Pietras K, Sjöblom T, Rubin K, Heldin CH, Ostman A: PDGF receptors as cancer drug targets. Cancer Cell 2003, 3:439-443.

36. Oertel S, Krempien R, Lindel K, Zabel A, Milker-Zabel S, Bischof M, Lipson KE, Peschke P, Debus J, Abdollahi A, Huber PE: Human Glioblastoma and Carcinoma Xenograft Tumors Treated by Combined Radiation and Imatinib (Gleevec $\left.{ }^{\odot}\right)$. Strahlenther Onkol 2006, I 82:400-7.

37. Abdollahi A, Lipson KE, Han X, Krempien R, Trinh T, Weber KJ, Hahnfeldt P, Hlatky L, Debus J, Howlett AR, Huber PE: SU54I6 and SU6668 attenuate the angiogenic effects of radiationinduced tumor cell growth factor production and amplify the direct anti-endothelial action of radiation in vitro. Cancer Res 2003, 63:3755-3763

38. Huber PE, Bischof M, Jenne J, Heiland S, Peschke P, Saffrich R, Gröne $\mathrm{HJ}$, Debus J, Lipson KE, Abdollahi A: Trimodal cancer treatment: beneficial effects of combined antiangiogenesis, radiation, and chemotherapy. Cancer Res 2005, 65:3643-55.

39. Bisch of M, Abdollahi A, Gong P, Stoffregen C, Lipson KE, Debus JU, Weber KJ, Huber PE: Triple combination of irradiation, chemotherapy (pemetrexed), and VEGFR inhibition (SU5416) in human endothelial and tumor cells. Int J Radiat Oncol Biol Phys 2004, 60:1220-1232

40. Abdollahi A, Griggs DW, Zieher H, Roth A, Lipson KE, Saffrich R, Gröne HI, Hallahan DE, Reisfeld RA, Debus J, Niethammer AG, Huber PE: Inhibition of integrin survival signaling enhances antiangiogenic and antitumor response of radiation. Clin Cancer Res 2005, I I:6270-9.

4I. Thilmann C, Nill S, Tücking T, Höss A, Hesse B, Dietrich L, Bendl R, Rhein B, Häring P, Thieke C, Oelfke U, Debus J, Huber P: Correction of patient positioning errors based on in-line cone beam CTs: clinical implementation and first experiments. Radiat Oncol 2006, 24; I: 16.

42. Münter MW, Schulz-Ertner D, Hof H, Nikoghosyan A, Jensen A, Nill $S$, Huber $P$, Debus J: Inverse planned stereotactic intensity modulated radiotherapy (IMRT) in the treatment of incompletely and completely resected adenoid cystic carcinomas of the head and neck: Initial clinical results and toxicity of treatment. Radiat Oncol 2006, 6(I): I7

43. Kerkelä R, Grazette L, Yacobi R, Iliescu C, Patten R, Beahm C, Walters B, Shevtsov S, Pesant S, Clubb FJ, Rosenzweig A, Salomon RN, Van Etten RA, Alroy J, Durand JB, Force T: Cardiotoxicity of the cancer therapeutic agent imatinib mesylate. Nat Med 2006, 2:908-16.

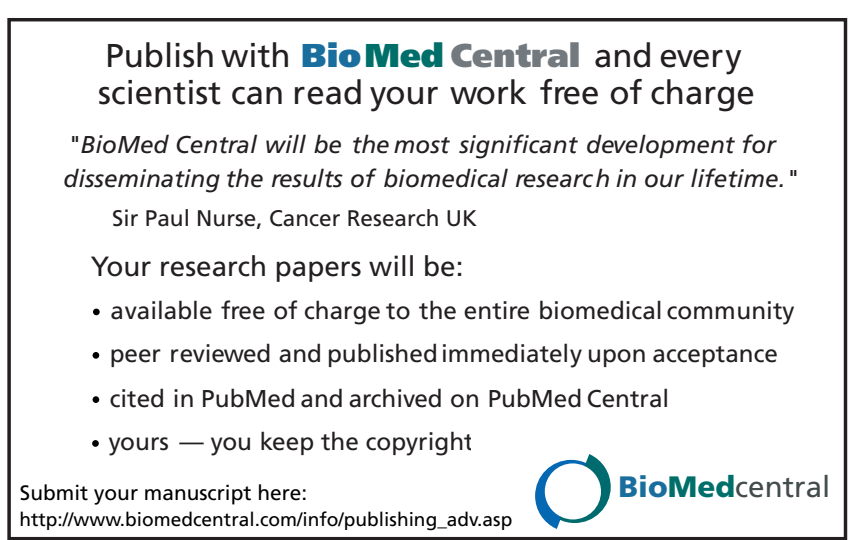

